\title{
THE
}

\section{Interventions During Labor and Birth in the United States: A Qualitative Analysis of Women's Experiences}

\author{
Alana M. Bibeau \\ University of Rhode Island, abibeau@uri.edu
}

Follow this and additional works at: https://digitalcommons.uri.edu/soc_facpubs

\section{The University of Rhode Island Faculty have made this article openly available.}

Please let us know how Open Access to this research benefits you.

This is a pre-publication author manuscript of the final, published article.

Terms of Use

This article is made available under the terms and conditions applicable towards Open Access

Policy Articles, as set forth in our Terms of Use.

\section{Citation/Publisher Attribution}

Bibeau, Alana M. "Interventions during labor and birth in the United States: a qualitative analysis of women's experiences." Sexual \& Reproductive Healthcare, vol. 5, no. 4, 2014, pp. 167-173.

http://dx.doi.org/10.1016/j.srhc.2014.10.003

Available at: http://dx.doi.org/10.1016/j.srhc.2014.10.003

This Article is brought to you for free and open access by the Sociology \& Anthropology at DigitalCommons@URI. It has been accepted for inclusion in Sociology \& Anthropology Faculty Publications by an authorized administrator of DigitalCommons@URI. For more information, please contact digitalcommons-group@uri.edu. 
Interventions During Labor and Birth in the United States:

A Qualitative Analysis of Women's Experiences

\author{
Alana M. Bibeau, Ph.D. \\ Department of Sociology \\ University of Virginia \\ Randall Hall 101 \\ 130 Ruppel Drive \\ P.O. Box 400766 \\ Charlottesville, VA $22904^{*}$
}

* Permanent Address: Department of Sociology \& Anthropology, University of Rhode Island, 507 Chafee Building, 10 Chafee Road Kingston, RI 02881. Email address: abibeau@mail.uri.edu 


\section{Abstract}

Objective: To explore and describe hospital-birthing women's understandings of and experiences with interventions during labor and birth.

Methods: Qualitative data was collected as part of a larger ethnographic study of childbirth in the United States. The grounded theory method was employed to analyze interviews with 59 women from three states who had recently given birth in hospitals with physicians or certified nurse-midwives in attendance.

Results: Four themes emerged from the data. The themes safety/risk and provider match, described women's expectations regarding intervention and their interactions with providers. A third theme addressed how women experienced interventions and their perceptions of control over decision-making. A final theme characterized women's satisfaction with maternity care.

Conclusions: Women who received interventions expressed varying levels of comfort or apprehension associated with both expectations of maternity care and provider match. Women whose expectations matched those of the provider reported more positive experiences. Regardless of provider match, women expressed ambivalence about the use of interventions and confusion over their appropriate place. Women's ability to make sense of interventions was related to how well they navigated a complicated and bureaucratic maternity system. Increasing attention needs to be paid to the impact of these factors on women's perceptions of care during pregnancy and childbirth.

Key Words: Intervention, Pregnancy, Birth, Qualitative Research, United States 


\section{Introduction}

Recent data from the World Health Organization shows American women are more likely to die in childbirth than they were two decades ago, making the U.S. one of only eight countries in the world where the risks from childbirth have risen in the past generation [1]. Despite advancements in prenatal care, access to medical technology, and frequent intervention in the labor process, the 2013 cesarean birth rate remained steady at 32.8\%, representing a nearly 60\% increase between 1996 and 2009 with no decrease in rates of maternal or neonatal morbidity or mortality $[2]$.

In a February 2014 consensus statement, the American College of Obstetricians and Gynecologists and the Society for Maternal-Fetal Health urged maternity care providers to reduce the primary cesarean birth rate, in part by utilizing more effective, low-risk tools to improve birth outcomes and by reducing unnecessary interventions [3]. Results from the Listening to Mothers III survey show that women giving birth in the U.S. routinely experience induction, augmentation, episiotomy, epidural or spinal analgesia, or other interventions during labor and childbirth - procedures that are inconsistent with best evidence and may increase the likelihood of cesarean birth [4]. Women also report troubling interactions with care providers, including pressure to agree to interventions they do not fully understand, and vastly underutilize low-risk options during pregnancy and childbirth such as seeking out midwifery care or the continuous labor support provided by doulas $[3,4,5]$. 
Survey data indicate that American women prefer minimal intervention in the birth process, but seldom experience this within the confines of a maternity care system that fails to promote evidence-based care. Despite this gap between preference and practice, the majority of American women indicate they have complete trust in their care providers, with $80 \%$ of women rating their care providers as "very trustworthy" or "completely trustworthy," a contradiction that is as pervasive as it is puzzling [4].

Studies in the U.S. [5-8] have explored the changing culture of American medicine and its impact on clinical practice for pregnant women and their care providers. Research demonstrates that high rates of Cesarean birth are tied to a medical culture that avoids risk and embraces predictability with the goal of perfect babies $[5,6]$. This discomfort with uncertainty and emphasis on the minimization of risk means that most hospitals are not set up to accommodate normal physiologic birth $[7,8]$. Additionally, research has found that malpractice premiums were positively associated with rates of cesarean section and primary cesarean section, and negatively associated with VBAC rates, making litigation a powerful force shaping U.S. maternity care [9].

International studies [10-12] have reported on the significance of women's beliefs about birth in shaping their experiences. Women with negative beliefs saw interventions as a way to manage what they perceived to be a frightening and painful ordeal, while women with positive beliefs often viewed interventions critically [10]. These studies also provide evidence that fear of labor and birth is a significant factor affecting women's use of interventions, but one that can be 
mediated through positive interactions with care providers [11]. Less is qualitatively known about how American women understand and experience interventions, or what factors effect their perceptions, making this population ripe for further qualitative investigation.

Changes in how women give birth in the U.S. today are not simply medical, but structural - brought about by enormous social, economic, legal, and political changes that have taken place over the last century. There is a long tradition in both the medical and sociological literatures conceptualizing the changes introduced by what scholars have identified as the "risk society" [13-16]. While in the medical view, risk is understood as an inevitable, objective reality that can be calculated and managed - the proverbial disaster waiting to happen - for sociologists, the individualization of risk is characterized by a loss of tradition and trust and by institutional instability $[13,14,17]$. In such a society we have become "disembedded" from tradition and compelled to make decisions and manage risks, untethered [13].

While some researchers have argued that the medicalization of pregnancy and birth compel women to participate in a technological imperative they do not fully understand $[18,19]$, others have argued that pregnancies in the risk society have developed into "planning projects" requiring an inordinate amount of attention and work on the part of pregnant women $[20,21]$. In teasing out some of the ways that women reflexively negotiate intervention use in the risk society, the research presented here builds upon these findings and reveals that while the rhetoric of safety, choice and control dominates mothers' understandings of pregnancy and 
birth, almost all respondents expressed deep anxiety about the place of interventions and indicated ambivalence about the extent to which technology has come to dominate the birth process.

These findings contribute to the existing literature by unpacking some of the ways the U.S. maternity care system and attendant perception of risk shape how women make decisions about pregnancy and birth - what they consent to and why, as well as the nature of their consent. Pregnant women in the risk society understand that they are expected, as autonomous beings, to make the right calculations, but they are also deeply afraid of making the wrong ones.

\section{Methods}

Qualitative, in-depth interviews were conducted with 59 women in the three U.S. states of Rhode Island, Virginia, and Washington who had given birth in hospitals with licensed care providers in attendance (Table 1). Additional data were collected from women who gave birth at home and in freestanding birth centers as part of a larger ethnographic project; separate manuscripts detailing those findings are in preparation. Maximum-variation sampling of states was used in an effort to be more representative of the U.S. population as a whole. Instead of seeking representativeness through equal probability, such a sample includes a range of extremes. The three states selected were geographically diverse and represented three different positions on the continuum of legal climates for birth in the U.S.

Washington has one of the highest rates of out-of-hospital births in the U.S., has been licensing non-nurse midwives since the 1980s, and both public and private 
Table 1

Demographic characteristics of sample.

\begin{tabular}{|c|c|c|}
\hline Characteristic & Number & Percent \\
\hline \multicolumn{3}{|l|}{ State } \\
\hline Rhode Island & 25 & 42.37 \\
\hline Virginia & 24 & 40.68 \\
\hline Washington & 10 & 16.95 \\
\hline \multicolumn{3}{|l|}{ Race/Ethnicity } \\
\hline White & 44 & 74.58 \\
\hline Black & 5 & 8.48 \\
\hline Asian/Pacific Islander & 7 & 11.86 \\
\hline Hispanic & 3 & 5.08 \\
\hline \multicolumn{3}{|l|}{ SES } \\
\hline Middle or Upper-Middle & 40 & 67.8 \\
\hline Lower-Middle or Working & 18 & 30.51 \\
\hline Working Poor & 1 & 1.69 \\
\hline \multicolumn{3}{|l|}{ Parity } \\
\hline Primiparous & 44 & 72.89 \\
\hline Multiparous & 16 & 27.11 \\
\hline \multicolumn{3}{|l|}{ Birth Attendant } \\
\hline Physician & 49 & 83.05 \\
\hline Certified Nurse-Midwife & 10 & 16.95 \\
\hline
\end{tabular}

insurance programs cover the cost of births regardless of setting. In addition to hospitals and homes, there are hospital-affiliated and freestanding birth centers throughout the state.

Rhode Island has one of the strictest and most prohibitive laws in the country, recognizing only physicians and nurse-midwives as birth attendants. ${ }^{\dagger}$

While home birth is not illegal, there were no care providers attending home births

† Rhode Island also recognizes Certified Midwives (CMs), health professionals who receive formal midwifery training and certify through the American Midwifery Certification Board, the governing body for nurse-midwives. There are currently no practicing CMs in the state. 
during the period of time in which data were collected. There are no freestanding birth centers, so the default option for most women is birth in the universityaffiliated maternity hospital or in one of the smaller, community hospitals. Those women who choose home births have long relied on the services of midwives from the neighboring states of Massachusetts and Connecticut who "cross the border" to attend these births.

Virginia's legal climate falls somewhere in the middle of the continuum. Virginia began licensing non-nurse midwives in 2005; before this time, non-nurse midwives who attended births were practicing illegally, though there was an active underground home birth movement prior to the passage of this legislation.

Freestanding birth centers exist in some of the more highly populated areas of the state, though the majority of women continue to give birth in hospitals.

Approval for the project was granted through the Institutional Review Board at the University of Virginia and written informed consent was obtained from all participants before beginning interviews. The consent form provided participants with information about the purpose of the research, time commitment involved, risks and benefits to participating in the study, and their right to withdraw at any time.

Face-to-face, semi-structured interviews consisted of several broad, openended questions designed to encourage and support participants' reflections while simultaneously maximizing their control over participation in the study. Respondents focused on sharing their birth stories - beginning with finding out they were pregnant, moving through birth, and concluding with the postpartum period - 
and brought up issues that were salient to them. Even though the interviews were conversational, the researcher was particularly interested in how women managed the cultural work of pregnancy and birth. Specific questions that always came up included, "On what basis did you select your care provider?" "Did you have any tests during pregnancy?" "Did you do any preparation for birth?" and "What did you do when labor started?"

The respondents were selected using a snowball sample method, relying on referrals from initial contacts to generate additional subjects. When soliciting referrals, participants were encouraged to consider family members, friends, and co-workers and referrals were limited to three from each participant. To maximize variation, additional participants were recruited from a number of public sources, including hospital bulletin boards, birth center newsletters, coffee shops, church bulletins, libraries, new mother support groups, and hospital-sponsored and private childbirth education classes.

The semi-structured, in-depth interviews lasted an average of two hours. Interviews were digitally recorded, transcribed, coded, and analyzed using the grounded theory method originally developed by Glaser and Strauss [22, 23]. Information was stored on a personal computer and password protected. No names were attached to sources of information and pseudonyms were used. Once saturation was reached, the interviews were transcribed and the data were analyzed.

A preliminary review of the transcriptions was conducted and an attempt was made to engage in conceptual ordering of the data into categories through the 
use of microanalysis [22]. This close reading of the transcriptions assisted the researcher in generating preliminary categories and suggested potential connections and relationships among the categories that emerged from the data [22]. Microanalysis of the transcribed interviews involved the actual data collected from the participants (i.e., their interpretations of their experiences), as well as the researcher's own observations and interpretations of these events and experiences in an effort to illuminate various properties and dimensions of the data. The next step in the analytic process was memoing, a process of elaborating on the coding categories that emerged from a close reading of the data [24].

\section{Results}

Of the 59 participants, $81 \%(n=48)$ experienced at least one intervention during labor and birth and 67\% $(n=40)$ experienced more than three. The most common types of interventions reported by women during in-depth interviews were, in order of frequency: continuous electronic fetal monitoring, IV, epidural anesthesia, artificial rupture of membranes, induction or augmentation of labor with synthetic oxytocin, episiotomy, and vacuum/forceps delivery. Eighteen women in the sample experienced Cesarean birth (Table 2).

Four major themes were identified from the analysis: (1) safety and the minimization of risk (2) the overall "match" between the participant's expectations of intervention and the provider's use of interventions during labor; (3) control over decision-making regarding intervention use; and (4) satisfaction with maternity care. These themes will be discussed in terms of women's perceptions, reactions, and preferences regarding intervention. 
Table 2

Frequency of intervention.

\begin{tabular}{lcc}
\hline $\begin{array}{c}\text { Most Frequent } \\
\text { Interventions }\end{array}$ & Total & Percent \\
\hline Continuous EFM & 52 & 88.14 \\
IV & 51 & 86.44 \\
Epidural anesthesia & 50 & 84.75 \\
AROM & 44 & 74.58 \\
Synthetic Oxytocin & 37 & 62.71 \\
Episiotomy & 21 & 35.59 \\
Cesarean & 18 & 30.51 \\
Vacuum/forceps & 6 & 10.17 \\
\hline
\end{tabular}

\section{Safety versus risk}

In every interview, the safety of hospitals was mentioned, suggesting the centrality of this concept in the construction of meaning surrounding birth. Even when they were vague about their definitions of the concept of safety, respondents were very quickly able to respond to questions about birth setting. Examples of these responses included:

"I wanted the stability and the safety net that you have in a hospital. You know that there's medical equipment there if it's needed." [Lilly]

"Giving birth is too much of a traumatic experience. You obviously want to be safe. You want to have that medical intervention there if it is necessary." [Bethany]

"Birth happens in hospitals. It's just safer... it's an institution. And you hope they're medical professionals that know what they are doing." [Katrina] 
The argument that hospitals are the safest place to be and that physicians are the experts in whom pregnant women place their trust is one that was heard repeatedly. Respondents consistently reported "doing whatever the [birth attendant] wanted" to ensure the safety of their unborn child.

Because safety played such a central role in their descriptions of childbirth, participants were asked to elaborate on what exactly safety meant to them. They described three things when prompted to deepen their responses: (1) the risks they perceived were inherent in the birth process; (2) the need to be prepared for the inevitability of these risks; and (3) the need to do everything possible to minimize them. For $83 \%$ of participants $(n=49)$ this included the possibility of interventions.

"The risk of something going wrong is really high. And so you need to recognize for yourself, 'Having a baby is not something that I can safely do without medical intervention."' [Kat]

"I felt better being able to look at the monitor, hearing how dilated I was, knowing the contractions were working. But it's just the way it is, just to be on the safe side, just in case anything bad happens and to make sure you're doing everything you can to prevent that." [Heidi]

"You kind of have a gauge of what your threshold is for certain risks and then you think about who and what can minimize them." [Stacey] While at first glance these respondents seem to focus on the medical risks involved in giving birth, a deeper analysis reveals that they are actually describing the individualization of risk - and the extent to which they view themselves as responsible for recognizing risk and doing "everything [they] can" to eliminate it. This more nuanced analysis tells a different story, where women themselves bear the responsibility for assuming and understanding the risks involved in giving birth and are obligated to act. While being in a hospital and following "doctor's orders" 
are seen as critical to minimizing risk, hospitals and care providers are better understood as tools pregnant women utilize in their efforts to manage risk.

\section{Provider match}

A second theme that emerged from the data was the overall "match" between the participant's expectations regarding intervention use and her care provider's approach to managing the labor process. When women expected medical interventions and their care provider intervened, there was a general sense of reassurance. The same was true for women who wanted to avoid intervention, when they felt that their care providers took a more hands-off approach. Some women believed that interventions increased their risks and sought midwifery care as a way to avoid them. Nearly $65 \%(\mathrm{n}=38)$ believed their provider's approach to managing labor and birth matched their expectations.

"I was completely confident in my doctor. My [obstetrics practice] had six different [doctors] there, and I liked all of them, they were very good. At one point [her obstetrician] said, 'I'd like to use a vacuum, we need to get this baby moving along and it's stuck right now. This is just for repositioning, it's not high pressure.' Well he did, he used the vacuum. And [the baby] was healthy and so I'm just really relieved that I had such good care." [Kathleen]

"The best of all possible worlds was having a midwife at the [inhospital] birth center. I trusted that she knew my wishes and was going to respect the integrity of the birth. I don't think that would have been true with the OB [who attended her first birth] because it just wasn't a good fit." [Anna]

While some mothers expressed comfort in knowing they and their care providers were on the same page, almost $36 \%$ of participants $(n=21)$ reported feeling "disconnected" and expressed confusion or disappointment when their expectations for birth did not align with the way their care providers practiced. 
"The doctor was like, 'Oh, and if you have a C-section we will put your incision right here,' and was showing me where she would make an incision on me for a C-section. I was probably, you know, only 3 months along. It's just... it was odd." [Julia]

"I wanted to have a drug-free birth. I was terrified of undergoing a surgical procedure, but [the doctor] kept telling me to think about [the] baby. It just seemed like...like maybe it would have been fine if they had left everything alone." [Andrea]

Respondents reported choosing care providers to serve as their advocates in an institutional system that they had little knowledge of or control over. The deep knowledge that would make such advocacy possible goes beyond health history and status updates; it also requires time spent getting to know patients and understanding their life histories, social circumstances, and personal preferences. The average time spent with care providers during a typical prenatal office visit was ten minutes for those respondents who saw obstetricians and forty-five minutes for those respondents who saw midwives:

"The nurse, of course, would always come in first for pre-natal stuff, to measure blood pressure and test urine and check weight and all of that. That took the most time. Then I'd wait for the doctor. " [Kat]

"The appointments are pretty fast. It was like 15 minutes in and out of there. Check this, do that and then, 'Yeah, we'll see you in 2 weeks or a month."' [Lilly]

"It was always either my midwife or her partner at the appointments. No nurses, no waiting, no five minutes with the doctor, wham-bamthank-you-ma'am. You can trust them and you feel good and I think when you're trusting the person, you know, you start to trust the whole birth thing." [Phoebe]

"Every appointment was an hour. I remember my midwife asking me once, 'Have you been able to find maternity clothes that work for you? It's so much more than just in and out." [Kimberly] 
These examples illuminate differences in prenatal care under the medical and midwifery models. They also show how pregnant women, in an effort to make the "right" choices, increasingly place their trust in medical experts, as the burden of risk becomes ever more complicated and specialized. Since the ultimate responsibility for managing risk falls to pregnant women, they rely on practitioners who share their philosophies of care regarding how and when to intervene. That they also report spending little time with physicians prenatally, suggests that philosophical differences in managing risk might not make themselves apparent until labor begins.

\section{Control over decision-making}

Women utilized varied "strategies of action" [25] as they negotiated the competing cultural demands for (1) safety and (2) choice/control over the birth process, including requesting interventions, adapting to protocols, or resisting provider recommendations altogether, with mixed results. For one participant, Kathleen, the process of laboring and giving birth to her son felt as if they were "beyond [her] control" and because of that, she said, she was more than happy to give her doctor free reign to decide what needed to happen during her birth:

"I assumed [the doctor] knew what he was doing and when I asked questions, I definitely got the sense he thought [induction] was no big deal. So I really didn't worry, but I did question him a little, like, "Are you sure this is okay?" And I just felt like he had made up his mind and it wasn't my decision to make and I didn't want to push it." [Lisa]

The majority of respondents interpreted control as decision-making power. While some demonstrated control by stating preferences, selecting interventions, and asking questions about suggestions made by care providers, others did so by 
avoiding medical intervention they viewed as unnecessary and potentially disruptive.

"I told the midwives that we wanted to kind of have our own space. I talked about the things that I didn't want done and that I did want done. Everything about the birth was my decision." [Alix]

"Birth is about choice and if I choose to have an epidural, then I am still in control of my birth because I am in control of the decisionmaking that goes into it." [Irene]

Those women who reported being most dissatisfied with their birth experiences were those who felt that they had not been given choices and who felt they had very little control over the process. That was certainly Andrea's experience when, in her thirty-ninth week of pregnancy, her doctor informed her at a routine appointment that since she was already two centimeters dilated, she "might as well head over to the hospital and have that baby." She reluctantly consented to a Cesarean birth after experiencing interventions including IV, synthetic oxytocin, epidural anesthesia, AROM, internal monitoring, and a urinary catheter during the nine hours she spent laboring. When her son's Apgar scores were good, she asked her physician what had necessitated the Cesarean and was told, "I guess he just really wanted to be born."

Respondents, when they felt they were actively involved in choosing things related to their birth - care providers, birth locations, medical interventions reported having some sense of control over their birth experiences. When medical experts made these choices for them rather than with them, women felt that their birth experiences were beyond their control. 


\section{Overall satisfaction with maternity care}

Given the extent to which women view themselves as accountable for managing risk, it makes sense that $56 \%$ of respondents $(n=33)$, reported feeling satisfied with their maternity care. Even in the absence of a strong provider match, respondents hold themselves responsible since they were the ones who ultimately chose the doctor and hospital:

"I was angry for a long time after. It was disappointing to see how everything we had discussed went out the window once labor started. Now, I know I should have done my homework. I mean, I thought I had, but it was really just the tip of the iceberg." [Lisa]

"It was fine. It was nothing that I wanted or planned for, but I can't really blame [the $\mathrm{OB}]$. He sees all the high-risk cases and just didn't know what to do with me - low-risk, birth plan in hand. It was a comedy of errors, the whole thing." [Dottie]

Further, respondents who were disappointed frequently fell back on the trope of safety, suggesting that they sacrificed their own need for control over the experience for the safety of their baby:

"Overall I would say I was happy with my care. I definitely had more interventions than I would have liked, which was disappointing. And I definitely wasn't always the one calling the shots, there were some tense discussions. But, then I got a healthy baby. " [Irene]

"I went along with what the nurses were saying because they said it was best for [the baby]. It was very confusing....and it still is, what was medically needed and what was just...easier...for them. I felt like a nuisance every time I made a sound! But, she was perfect when she was born, so I guess I did something right." [Margaret]

Forty-four percent of women ( $n=26)$ expressed regret over the management of their births during interviews, even while justifying why induction, augmentation, episiotomy, epidural or spinal analgesia, or other interventions were necessary during their births. Some women were troubled by the physician's absence during 
their labors - showing up at the last minute to deliver their babies - and at the fact that in many practices the physician who happened to be on call when they went into labor was someone they had never met before. They described doctors as "absent," "disinterested," "authoritative," and "persuasive," holding a kind of "power" that led to feeling "dismissed," "secondary," and "out of [my] element."

About $17 \%$ of respondents $(n=10)$, described taking an active role in the birth process:

"I think just hearing that I was 10 centimeters [dilated], I thought, 'I guess it's okay to push any time now.' Every once in a while they would listen for the heartbeat and I remember my husband asked them, 'Well when can she start pushing?' and they said, 'Look at her, she is pushing now."' [Alix]

Of this group of respondents, all but two were attended by certified nurse-midwives. They reported feeling "respected" and "supported" by their caregivers and treated as if they themselves were the ones "in charge" during their labors. This distinction is important, because what these respondents convey through their stories is a feeling of ownership of the birth itself, rather than ownership over the management of birth.

\section{Discussion}

The analysis presented here is of data collected from a snowball sample of women who gave birth in hospitals in the three U.S. states of Rhode Island, Virginia and Washington. The qualitative, in-depth interviews were conducted as part of a larger, ethnographic study of childbirth in the U.S. Given the aim of the study, the findings suggest much more similarity than difference across states. Despite the varying status of midwives, a hospital culture of intervention exists that is 
independent of state locality. The findings further suggest that women's experiences with interventions during labor and birth are mixed.

Participants had varied preferences regarding intervention. Several themes indicated that women prioritized safety concerns related to their babies and therefore saw interventions as one way to minimize risk. They believed these risks were inherent in the labor and birth process and could be mediated through the use medical and technological interventions, even if these interventions represented a change in their expectations about how the birth would unfold. Other participants believed, as research shows, that interventions increased their risks and sought midwifery care as a way to avoid them [26, 27].

The role of evidence-based childbirth education classes is therefore an important issue for maternity health care professionals to consider, particularly since there is growing evidence to show that the majority of first-time mothers participate in such classes [4]. While attendance at childbirth preparation classes has been shown to decrease anxiety and fear surrounding birth, it has also been shown to have a negative impact on intervention use $[28,29]$. The findings reported here suggest that these classes could provide a potentially rich source of information about the actual (versus perceived) risks involved in birth for low-risk women, as well as the risks involved in the use of common medical interventions during labor.

Findings also show that women were reassured when their provider's philosophy of care and the management approach they utilized during labor aligned with women's own expectations. Women who expected routine interventions were 
reassured by providers who employed them. Likewise, women who hoped to avoid routine interventions felt betrayed by providers who casually intervened and expressed belated confusion over whether these interventions were truly medically necessary.

Previous research has shown that the majority of American women view their maternity care provider as a "very valuable" source of information about pregnancy and birth [4]. The difficulty arises when patients and providers have divergent philosophical positions and expectations for interventions use and these differences are not made explicit prenatally $[30,31]$. The findings presented here confirm the significant role maternity health care providers play. The findings further suggest that maternity health care providers are uniquely positioned to encourage women to discuss their preferences and perceptions during prenatal care in an effort to uphold realistic expectations for both parties.

Findings also show that it is important for women that they are in control of the management of labor and birth, even when they acquiesce to provider recommendations they may not fully support. This supports quantitative research conducted by Fair and Morrison demonstrating that control during the birth process was an important predictor of birth satisfaction [32]. While an emphasis on safety is not unique to American women - or to American hospitals - notions of choice and control are uniquely central to American cultural values [33]. For the majority of participants choice often meant placing their trust - and their bodies - in the hands of a physician. For others it involved negotiating with care providers and advocating for a birth that minimized intervention to the extent possible in 
institutions where the standard policies and procedures that are in place are designed to minimize litigation, $[5,8]$.

Women described the difficulty inherent in balancing safety needs with the desire for control during the birth process, a difficulty that was compounded when care providers intervened in ways that were inconsistent with the participants' expectations. The findings suggest that women actively strive to balance these competing cultural demands, even as they express ambivalence regarding the use of interventions.

The study was limited to women living in three U.S. states, and for this reason, researchers should be cautioned against generalizations. The three states were chosen because they represented three different legal and political climates surrounding the licensing of birth attendants, thus impacting women's access to all available options. A main limitation of the study is that it only includes women who gave birth in hospitals. Additional data was collected from women who gave birth in freestanding birth centers and at home as part of the larger ethnographic study from which the data is derived. A manuscript detailing these findings is in preparation. Although the findings presented here describe the experiences of a relatively small sample, the inclusion of women from three states and the insights generated through in-depth interviews provide important information on how women in the U.S. understand interventions.

A potential drawback of qualitative studies is that results could be limited by the small snowball sample, however this limitation can be overcome if saturation of the data is achieved, as it was here. The ability to generalize findings is further 
limited because of the demographic profile of the participants who were largely, though not exclusively, white and middle-class. In the larger study, women who gave birth at home and birth centers were also over-sampled, which could have impacted the referral chain, though steps were taken to limit references.

\section{Conclusion}

This study extends the body of largely quantitative research on interventions during labor and birth by taking a deeper, qualitative look at U.S. women's understanding of and experiences with interventions during parturition. Women's experiences with interventions are nuanced and require us to consider the complex cultural relationship between safety/risk on one hand and choice/control on the other. This American cultural tension plays a largely ignored role in shaping the provision of maternity care in hospital settings.

Safety - through an attempt to minimize and prevent risk - serves as a cultural force that actively shapes our concept of health, our belief that perfection is possible, the relationships we develop with technology and intervention, and our understanding of consent. The individualization of risk obligates women to reflexively manage the risks of pregnancy and birth privately, disembedded from the traditional local networks that once lent their lives form and meaning and where midwives lived and worked side-by-side in communities with the women they served. The introduction of technology into the birth process means that women are confronted with often conflicting information concerning potential risks to the fetuses they carry and it is fully expected that they will respond to the these risks, whether perceived or actual. The burden of understanding those risks and making 
choices among an ever-increasing and specialized range of options leads to an increased reliance on medical expertise. In this sense, interventions are not simply something that happen to women, but practices in which women are - or can be actively engaged.

When women's expectations regarding the use of interventions aligned with the way their care providers practiced, they were more likely to see intervention, when and if it happened, as something that was medically indicated, and therefore beneficial, to themselves and their babies. In the current climate of high rates of interventions, these factors suggest several directions for future research.

Increasing attention needs to be paid to why women believe more technology automatically results in safer outcomes. Additionally, we need research that explores how not just the content but also the nature of maternity care impacts health and social outcomes. Finally, research needs to further explore the varying knowledge, tools, and expectations for care that women bring to the conversation about birth and how such factors impact their ability to advocate for safer, evidencebased maternity care within medical institutions. Within the context of maternity care, it is clear that there is a need for competent and respectful providers who value women as partners, understand their fears, provide evidence-based care, encourage the use of low-risk interventions like childbirth education and doula support, and facilitate authentic, informed choice for women.

\section{Acknowledgements}

The author would especially like to thank Jennifer M. Silva for her guidance and helpful feedback on the manuscript; Rae Lesser Blumberg, Sarah M. Corse, and 


\section{Allison J. Pugh for their support of the project; and four anonymous Sexual and Reproductive Healthcare reviewers.}

\section{References}

[1] The Lancet Global Health, Early Online Publication, 6 May 2014. doi:10.1016/S2214109X(14)70227-X

[2] Martin JA, Hamilton BE, Osterman JK, et al. Births: Final data for 2012. National vital statistics reports; vol 62 no 9. Hyattsville, MD: National Center for Health Statistics. 2013.

[3] American College of Obstetricians and Gynecologists and the Society for Maternal-Fetal Medicine. Safe prevention of the primary cesarean delivery. Obstetric Care Consensus No. 1. American College of Obstetricians and Gynecologists. Obstet Gynecol 2014;123:693-711.

[4] Declercq ER, Sakala C, Corry MP, Applebaum S, Herrlich A. Listening to Mothers SM III: Pregnancy and Birth. New York: Childbirth Connection, May 2013.

[5] Downe, S. Engaging with the concept of unique normality in childbirth. Br J Midwifery 2006; 14(6): 352-356.

[6] Morris, T. Cut It Out: The c-section epidemic in America. New York: NYU Press; 2013.

[7] Kennedy HP. The Problem of Normal Birth. J Midwifery Wom Heal 2010;55(3):199-201.

[8] Goer RE, Romano AM. Optimal care in childbirth: The case for a physiologic approach. Seattle, WA: Classic Day; 2012.

[9] Yang YT, Mello MM, Subramanian SV, Studdert DN. Relationship Between Malpractice Litigation Pressure and Ceserean Section and Vaginal Birth After Cesarean Section. Med Care 2009; 47(2): 234-242.

[10] Stoll K, Hall W. Attitudes and Preferences of Young Women With Low and High Fear of Childbirth. Qual Health Res 2013;23(11):1495-1505.

[11] Klomp T, Manniën J, de Jonge A, Hutton EK, Lagro-Janssen ALM. What do midwives need to know about approaches of women towards labour pain management? A qualitative interview study into expectations of management of labour pain for pregnant women receiving midwife-l led care in the Netherlands. Midwifery 2014; 30(4):432-428. 
[12] Bayes S, Fenwick J, Hauck Y. A Qualitative analysis of Women's Short Accounts of Labour and Birth in a Western Australian Public Tertiary Hospital. J Midwifery Wom Heal 2008;53(1): 53 61.

[13] Giddens A. The Consequences of Modernity. Cambridge: Polity Press; 1990

[14] Giddens, A. Modernity and Self-Identity: self and society in the late modern age. Stanford, CA: Stanford University Press; 1991.

[15] Lupton D. Medicine as Culture: illness, disease, and the body in western societies. London: Sage; 1993.

[16] Skolbekken JA. The risk epidemic in medical journals. Soc Sci Med 1995 Feb; 40(3): 291-205.

[17] Beck U. Risk Society: towards a new modernity. London: Sage; 1992.

[18] Davis-Floyd R. Culture and Birth" the technocratic imperative. Int J Childbirth Educ 1994, 9(2):67

[19] Katz Rothman B. Genetic Maps and Human Imaginations: the limits of science in understanding who we are. New York: WW Norton; 1998.

[20] Beck U \& Beck-Gernsheim E. Individualization. London: Sage; 2002

[21] Possamai-Indesedy A. Confining Risk: Choice and responsibility in childbirth in a risk society. Health Sociol Rev 2006, 15(4): 406-414.

[22] Glaser B, Strauss A. Discovery of Grounded Theory. Chicago: Aldine; 1967.

[23] Strauss A. Qualitative Analysis for Social Scientists. Cambridge, UK: University of Cambridge; 1987.

[24] Strauss A and Corbin J. Basics of Qualitative Research: Techniques and Procedures for Developing Grounded Theory, 3rd ed. Thousand Oaks, CA: Sage Publications. 2008.

[25] Swidler A. Culture in Action: Symbols and Strategies. Am Sociol Rev1986;51(2):273-286.

[26] Romano AM, Lothian JA. Promoting, protecting, and supporting normal birth: A look at the evidence. J Obstet Gynecol Neonatal Nur 2008; 37(1): 94-105.

[27] Kennedy HP, Shannon MT. Keeping birth normal: Research findings on midwifery care during childbirth practice. J Obstet Gynecol Neonatal Nur 2003; 33(5): 554-560.

[28] Ferguson,F, Davis D, Browne J. Does antenatal education affect labour and birth? A structured 
review of the literature. Women Birth 2013;26(1):e5-e8.

[29] Lothian J. Listening to Mothers II: Knowledge, decision-making, and attendance at childbirth education classes. J Perinat Educ 2007; 16(4): 62-67.

[30] Malacrida C, Boulton T. The best laid plans? Women's choices, expectations and experiences in childbirth. Health 2014; 18(1): 41-49.

[31] Soliday E. Childbirth in a technocratic age: Documentation of women's expectations and experiences. Amherst, NY: Cambria Press. 2012.

[32] Fair C, Morrison T. The relationship between prenatal control, expectations, experienced control, and birth satisfaction among primiparous women. Midwifery 2012; 28(1): 39-44.

[33] Rutherford M. Authority, Autonomy, and Ambivalence: Moral Choice in Twentieth-Century Commencement Speeches. Sociol Forum 2004;19(4): 583-610. 